\title{
PARADOXICAL FUNCTIONS ON THE INTERVAL
}

\author{
VICTOR JIMÉNEZ LÓPEZ \\ (Communicated by Andrew M. Bruckner)
}

\begin{abstract}
In this paper it is shown that any expanding with Lipschitz derivative function $f$ has a contradictory behaviour from the point of view of chaos in the sense of $\mathrm{Li}$ and Yorke. On the one hand it cannot generate scrambled sets of positive Lebesgue measure. On the other hand the two-dimensional set $\mathrm{Ch}(f)$ including the pairs $(x, y)$ such that $\{x, y\}$ is a scrambled set of $f$ has positive measure. In fact, both the geometric structure (almost everywhere) and measure of $\mathrm{Ch}(f)$ can be explicitly obtained.
\end{abstract}

\section{INTRODUCTION AND STATEMENT OF THE RESULTS}

Over the last few years an important amount of work has been done about the notion of chaos such as it was defined by Li and Yorke in [15] (see also [10] and [14]). Throughout the paper $\mathscr{C}(I)$ will denote the set of continuous functions $f: I \rightarrow I$, where $I$ is a compact real interval.

Definition 1.1. Let $f \in \mathscr{C}(I)$. Suppose that there exists $S \subset I$ with at least two elements such that for any $x, y$ belonging to $S, x \neq y$, and any periodic point $p$ of $f$

$$
\begin{aligned}
& \limsup _{n \rightarrow \infty}\left|f^{n}(x)-f^{n}(y)\right|>0, \\
& \liminf _{n \rightarrow \infty}\left|f^{n}(x)-f^{n}(y)\right|=0, \\
& \limsup _{n \rightarrow \infty}\left|f^{n}(x)-f^{n}(p)\right|>0 .
\end{aligned}
$$

Then $S$ is called a scrambled set (of $f$ ) and $f$ is said to be chaotic (in the sense of Li and Yorke).

It is well known (see [7] or [10]) that if $f$ has a periodic point of period not a power of 2 (here we include 1 as a power of two) then it is chaotic. Nevertheless this chaos can essentially be "unobservable". Consider $f(x)=a-1-a x^{2}$, where $a(a-1)=1.75487 \ldots$ is the root of the equation $1-x(x-1)^{2}=0$. For this function, 0 is a periodic point of period 3, so it is chaotic. On the other hand almost every $x \in[-1,1]$ (with respect to the Lebesgue measure) is attracted by the orbit of 0 (see [8]).

Received by the editors August 26, 1990 and, in revised form, May 13, 1992.

1991 Mathematics Subject Classification. Primary 26A18, 58F13; Secondary 58F15. 
Then a rather natural question is whether there can exist chaotic functions possessing a scrambled set of positive measure. In what follows, $\lambda$ and $\lambda^{2}$ will respectively denote one-dimensional and two-dimensional Lebesgue measure.

Definition 1.2. Let $f \in \mathscr{C}(I)$. Then $f$ is said to be strongly chaotic from the point of view of measure (or, more briefly, sm-chaotic) if it has a measurable scrambled set of positive measure.

A first partial answer to this problem was given by Smital [20], where he proves that the function $f:[0,1] \rightarrow[0,1]$ given by $f(x)=1-|2 x-1|$ has a scrambled set of outer measure 1 . However, for expanding functions we have the following (we say that $f \in \mathscr{C}(I)$ is expanding-we also put $f \in$ $\mathscr{E}(I)$-if it is piecewise monotonic and there exists a constant $\kappa_{f}>1$ such that $|f(x)-f(y)| \geq \kappa_{f}|x-y|$ for every pair of points $x, y$ belonging to any interval on which $f$ is monotonic):

Proposition 1.3. Let $f \in \mathscr{E}(I)$. Then it cannot be sm-chaotic.

Proof. Suppose $S$ is a measurable scrambled set with $\lambda(S)>0$. Since $f^{n} \mid S$ is one-to-one for any $n$, we have $\lambda\left(f^{n}(S)\right) \geq \kappa_{f}^{n} \lambda(S)$, so $\lambda\left(f^{k}(S)\right)>\lambda(I)$ for some $k$, which is impossible.

The first examples of sm-chaotic functions were simultaneously found by Smítal [21] and Kan [13]. Afterward Janková and Smital (see [10] and [22]) proved that, in fact, any chaotic function is topologically conjugate to an smchaotic function. Maps with scrambled sets of full measure are also known; see Misiurewicz [17] and Bruckner and $\mathrm{Hu}$ [3]. All the above functions are nondifferentiable; a $C^{\infty}$ sm-chaotic function is given in [11].

The problem with these maps (even the smooth one) is that they all are highly sophisticated. Indeed, there are no known piecewise linear or analytic smchaotic functions. Moreover, to identify sm-chaoticity with "large" chaos can be misleading. In fact, $f(x)=1-|2 x-1|$ is not sm-chaotic but exhibits sensitive dependence with respect to initial conditions and possesses an invariant ergodic measure (Legesgue measure itself). Thus $f$ shows in some sense a "paradoxical" behaviour from the point of view of measure. To deal with this situation, the following notion will be useful.

Definition 1.4. Let $f \in \mathscr{C}(I)$. Define $\operatorname{Ch}(f) \subset I \times I$ as the set of points $(x, y)$ holding

$$
\begin{gathered}
\limsup _{n \rightarrow \infty}\left|f^{n}(x)-f^{n}(y)\right|>0, \\
\liminf _{n \rightarrow \infty}\left|f^{n}(x)-f^{n}(y)\right|=0,
\end{gathered}
$$

and for any periodic point $p$ of $f$

$$
\limsup _{n \rightarrow \infty}\left|f^{n}(x)-f^{n}(p)\right|>0, \quad \limsup _{n \rightarrow \infty}\left|f^{n}(y)-f^{n}(p)\right|>0 .
$$

Then $f$ is said to be empirically chaotic if $\lambda^{2}(\mathrm{Ch}(f))>0$.

Notice that $\operatorname{Ch}(f)$ is always measurable.

Remark 1.5. The idea of passing to the square, in a different context, was suggested by Lasota and has been used by Piórek [18], Snoha [19], and Gedeon [6]. In fact, they work with the set $\mathrm{Ch}^{*}(f)$ of points verifying $(\mathrm{CH} 1)$ and $(\mathrm{CH} 2)$. 
Note that, if $S$ is a set holding (S1) and (S2) in Definition 1.1, then by taking off from it at most an adequately chosen point, it also verifies (S3). On the other hand there exist examples of functions $f$ holding $\lambda^{2}\left(\mathrm{Ch}^{*}(f)\right)>0$ but $\lambda^{2}(\mathrm{Ch}(f))=0$ (see [12]). If $f$ is expanding then $\mathrm{Ch}^{*}(f) \backslash \mathrm{Ch}(f)$ is countable and so $\lambda^{2}\left(\mathrm{Ch}^{*}(f)\right)=\lambda^{2}(\mathrm{Ch}(f))$.

Definition 1.6. Let $f \in \mathscr{C}(I)$. Then $f$ is said to be paradoxical if it is empirically chaotic but not sm-chaotic.

In [20] it is implicitly proved that $f(x)=1-|2 x-1|$ is paradoxical, with $\mathrm{Ch}(f)$ possessing full measure. This conclusion was generalized by Gedeon [6] to the family of full piecewise linear functions. Here a function $f:[a, b] \rightarrow$ $[a, b]$ is called full piecewise linear if there exists a partition $a=a_{0}<a_{1}<a_{2}<$ $\cdots<a_{k}=b$ with $k>1$ such that $\left.f\right|_{\left[a_{i-1}, a_{i}\right]}$ is linear and $f\left(\left[a_{i-1}, a_{i}\right]\right)=[a, b]$ for any $i$.

The aim of this paper is to show that a rather big class of maps, namely, expanding with Lipschitz derivative, are paradoxical. It turns out that in these cases the geometric structure of $\mathrm{Ch}(f)$ can (almost everywhere) be explicitly described and its measure explicitly calculated.

Definition 1.7. Let $f \in \mathscr{C}([a, b])$. Then $f$ is said to be expanding with Lipschitz derivative if there exists a (minimal) partition $a=a_{0}<a_{1}<a_{2}<\cdots<$ $a_{k}=b$ and a constant $\kappa_{f}>1$ such that $f_{i}=\left.f\right|_{\left(a_{i-1}, a_{i}\right)}$ is differentiable for any $i$ with $\left(f_{i}\right)^{\prime}$ Lipschitz and verifying $\left|\left(f_{i}\right)^{\prime}(x)\right| \geq \kappa_{f}$ for any $x$. The points $a_{1}, a_{2}, \ldots, a_{k-1}$ are called singular.

We shall denote the set of expanding with Lipschitz derivative functions $f: I \rightarrow I$ as $\mathscr{L}(I)$.

Now we can state our main result.

Theorem 1.8. Let $f \in \mathscr{L}(I)$. Then there exists a family $\left\{I_{n}\right\}_{n}$ of subintervals of $I$ with pairwise disjoint interiors holding the following properties:

(i) $\sum_{n} \lambda\left(I_{n}\right)=\lambda(I)$.

(ii) Let $I_{k}, I_{l}$ belong to $\left\{I_{n}\right\}_{n}$. If there exists a nonnegative integer $m$ such that $f^{m}\left(I_{k}\right) \cap f^{m}\left(I_{l}\right) \neq \varnothing$, then

$$
\lambda^{2}\left(\mathrm{Ch}(f) \cap\left(I_{k} \times I_{l}\right)\right)=\lambda\left(I_{k}\right) \lambda\left(I_{l}\right)
$$

otherwise

$$
\lambda^{2}\left(\mathrm{Ch}(f) \cap\left(I_{k} \times I_{l}\right)\right)=0 .
$$

Remark 1.9. Indeed the proof of Theorem 1.8 provides us with more precise information on $\left\{I_{n}\right\}_{n}$, so every $f\left(I_{n}\right)$ is included in some $I_{m}$. Moreover, there exists a finite family $\left\{B_{i}\right\}_{i} \subset\left\{I_{n}\right\}_{n}$ (with $\left\{B_{i}\right\}_{i}$ the bitransitive intervals of $f$, see Definition 2.4) such that $f\left(\bigcup_{i} B_{i}\right) \subset \bigcup_{i} B_{i}$, and for every $I_{n}$ there exist some $B_{i}$ and $m \geq 0$ with $f^{m}\left(I_{n}\right) \subset B_{i}$. On the other hand, the relative position of the intervals $\left\{B_{i}\right\}_{i}$ can specifically be described as in [1].

Corollary 1.10. Let $f \in \mathscr{L}(I)$. Then it is paradoxical.

Proof. It immediately follows from Theorem 1.8 and Proposition 1.3.

Remark 1.11. Theorem 1.8 does not work if functions $f_{i}$ in Definition 1.7 are only $C^{1}$; see [12] for a counterexample. The same function also provides counterexamples for Lemmas 3.7, 3.8, and (with a slight modification) 3.5. Still, the question of whether every expanding function is paradoxical remains open. 


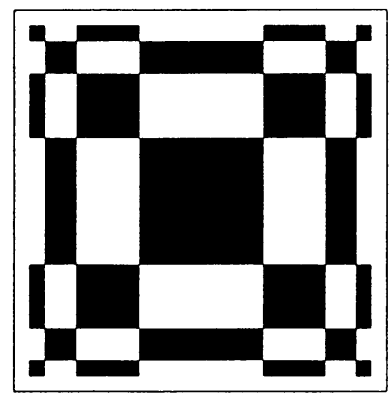

FIGURE 1

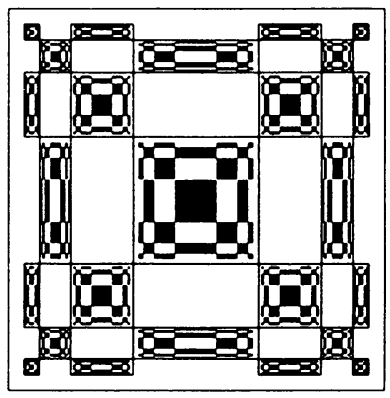

FIGURE 2

Corollary 1.12 (see Gedeon [6]). Let $f: I \rightarrow I$ be a full piecewise linear function. Then $\lambda^{2}(\mathrm{Ch}(f))=(\lambda(I))^{2}$.

Proof. Obvious, since for every subinterval $J$ of $I$ there exists some $k$ such that $f^{k}(J)=I$.

Corollary 1.13. There exist analytic paradoxical functions.

Proof. Consider the functions $f, g:[0,1] \rightarrow[0,1]$ respectively given by $f(x)=1-|2 x-1|$ and $g(y)=4 y(1-y)$, which are topologically conjugate through $h(x)=\sin ^{2}(\pi x / 2)$. Note that $(x, y) \in \operatorname{Ch}(f)$ if and only if $(h(x), h(y)) \in \mathrm{Ch}(g)$. Since $f$ is paradoxical and $h$ is smooth, we conclude that $g$ is also paradoxical.

Remark 1.14. In fact, from $\lambda^{2}(\mathrm{Ch}(f))=1$ (see Corollary 1.12), one can also obtain $\lambda^{2}(\mathrm{Ch}(g))=1$.

We finish this introductory section with an example illustrating Theorem 1.8. Namely, consider the family of functions $f_{a}:[0,1] \rightarrow[0,1]$ given by $f_{a}(x)=a(1-|2 x-1|) / 2$, where $a$ is a parameter belonging to $(1,2]$. Using some estimations from [23] one can describe $\mathrm{Ch}\left(f_{a}\right)$ in an effective way. For example, $\lambda^{2}\left(\mathrm{Ch}\left(f_{a}\right)\right)=1$ when $\sqrt{2} \leq a \leq 2$, while Figures 1 and 2 show the (almost everywhere) geometric structure of $\mathrm{Ch}\left(f_{a}\right)$ (painted in black) when $\sqrt[4]{2} \leq a<\sqrt{2}$ and $\sqrt[8]{2} \leq a<\sqrt[4]{2}$ respectively (the proportions have been distorted to increase the clarity of pictures). Moreover, the specific value of $\lambda^{2}\left(\mathrm{Ch}\left(f_{a}\right)\right)$ can be also calculated in all cases (see [12]).

\section{SOME PRELIMINARY LEMMAS ON TRANSITIVITY}

Before beginning the proof of Theorem 1.8 we recall some necessary results on transitivity that will be useful later.

Definition 2.1. Let $f \in \mathscr{C}(I)$. Then $f$ is said to be bitransitive if $f^{2}$ is (topologically) transitive.

Lemma 2.2 (see [2]). Let $f \in \mathscr{C}(I)$.

(i) If $f$ is bitransitive then $f^{n}$ is transitive for any positive integer $n$.

(ii) If $f$ is transitive then either $f$ is bitransitive or there exist $J, K$ closed subintervals of $I$ verifying $J \cup K=I, \operatorname{Int}(J) \cap \operatorname{Int}(K)=\varnothing$, and such that $f(J)=K$ and $f(K)=J$, being $\left.f^{2}\right|_{J},\left.f^{2}\right|_{K}$ bitransitive. 
Lemma 2.3 (see [4]). Let $f: I \rightarrow I$ be a bitransitive piecewise monotonic function. Then for any subinterval $J$ of $I$ there exists an integer $k$ such that $f^{k}(J)=I$.

In fact, we are mainly interested in intervals on which some iterate of $f$ behaves as a bitransitive function.

Definition 2.4. Let $f \in \mathscr{C}(I)$ and $J$ be a closed subinterval of $f$. We say that $J$ is bitransitive if there exists some $r \geq 1$ such that $\left.f^{r}\right|_{J}: J \rightarrow J$ is bitransitive.

We shall denote the union set of all bitransitive intervals of $f$ as $\mathscr{O}(f)$.

Lemma 2.5. Let $f \in \mathscr{C}(I)$.

(i) If $J$ is bitransitive then $f^{i}(J)$ is bitransitive for any $i$.

(ii) If $J \neq K$ are bitransitive then $\operatorname{Int}(J) \cap \operatorname{Int}(K)=\varnothing$.

Proof. (i) is immediate. For (ii), assume the contrary. Let $r, s$ be such that $\left.f^{r}\right|_{J}$ and $\left.f^{s}\right|_{K}$ are bitransitive. Since $f^{r s}(J \cap K) \subset J \cap K$, applying Lemima 2.2(i) we have $J=K$, a contradiction.

Remark 2.6. If $f$ is piecewise monotonic, from Lemma 2.5 it has a finite number of bitransitive intervals.

In the general case, of course, $\mathscr{O}(f)$ can be empty. Nevertheless this is not possible for expanding functions.

Lemma 2.7. Let $f \in \mathscr{E}(I)$. Then it has some bitransitive interval.

Proof. In [9] it is proved that if $f$ is expanding there exist an integer $r$ and a subinterval $J$ of $I$ such that $\left.f^{r}\right|_{J}: J \rightarrow J$ is transitive. This result together with Lemma 2.2(ii) implies Lemma 2.7.

\section{Proof of Theorem 1.8}

First of all let us see an outline with the main ideas in the proof. Key results are Proposition 3.1 and Lemma 3.3 (this last one simply allows us to consider our functions as "almost" piecewise linear). With them plus Lemma 2.7 we prove Lemma 3.5, which leads us to concentrate on bitransitive functions. If $f$ is bitransitive, Lemma 2.3 (and Proposition 3.1 and Lemma 3.3 again) imply that $f$ behaves in some sense as a full piecewise linear function, and from here it is relatively easy to prove Lemma 3.8 , which essentially finishes the proof.

Proposition 3.1. Let $f \in \mathscr{E}(I)$ and $J$ be a subinterval of $I$. Then there exist a constant $\varepsilon_{f}>0$ depending only of $f$ and a family $\left\{J_{n}\right\}_{n}$ of pairwise disjoint subintervals of $J$ holding the following properties:

(i) $\sum_{n} \lambda\left(J_{n}\right)=\lambda(J)$.

(ii) For every $J_{n}$ there exists some $k$ such that $\left.f^{k}\right|_{J_{n}}$ is monotonic and $\lambda\left(f^{k}\left(J_{n}\right)\right) \geq \varepsilon_{f}$.

Proof. It is not restrictive to suppose that $\kappa_{f}>2$ (if necessary we would replace $f$ by an adequate $f^{l}$ ). Take $\varepsilon_{f}>0$ small enough such that if $K$ is a subinterval of $I$ with $\lambda(K)<\varepsilon_{f}$ then it can contain at most a turning point of $f$. 
Let $\mathscr{J}_{m}$ for any $m$ be the set of maximal open subintervals of $J$ on which $f^{m}$ is monotonic. Inductively define $\mathscr{K}_{1}=\left\{K \in \mathscr{J}_{1}: \lambda(f(K)) \geq \varepsilon_{f}\right\}$ and

$$
\mathscr{K}_{m}=\left\{K \in \mathscr{J}_{m}: \lambda\left(f^{m}(K)\right) \geq \varepsilon_{f} \text { and } K \cap L=\varnothing \text { for any } L \in \bigcup_{i=1}^{m-1} \mathscr{K}_{i}\right\} \text {. }
$$

Then put $\left\{J_{n}\right\}_{n}=\bigcup_{m=1}^{\infty} \mathscr{K}_{m}$. Notice that all these intervals are pairwise disjoint while condition (ii) is trivially verified. With regard to (i), note that if $K \in \mathscr{J}_{m}$ but $\lambda\left(f^{m}(K)\right)<\varepsilon_{f}$ then $\left.f^{m+1}\right|_{K}$ has at most a turning point. Thus the set of intervals $K \in \mathscr{J}_{m}$ such that $K \cap L=\varnothing$ for any $L \in \bigcup_{i=1}^{m} \mathscr{K}_{i}$ has cardinality at most $r 2^{m-1}$, where $r$ is the number of pieces of monotonicity of $f$. Moreover, if $K$ is one of these intervals, then $\lambda(K)<\varepsilon_{f} / \kappa_{f}^{m}$. Hence,

$$
\lambda\left(J \backslash\left(\bigcup_{i=1}^{m} \bigcup_{K \in \mathscr{K}_{i}} K\right)\right)<\frac{r \varepsilon_{f} 2^{m-1}}{\kappa_{f}^{m}}
$$

and (i) follows.

Remark 3.2. Notice that if $f \in \mathscr{L}(I)$ we can choose the intervals $\left\{J_{n}\right\}_{n}$ with the additional property of not containing singular points.

Now we need an appropriate version of a classical Denjoy's lemma (see [5]). It is implicitly shown in [23], but no concrete reference can be suggested, so we give the proof here.

Lemma 3.3. Let $f \in \mathscr{L}(I), k$ be a positive integer, and $J$ be a subinterval of $I$. Suppose that the intervals $J, f(J), \ldots, f^{k-1}(J)$ do not contain singular points in their interiors. Then there exists a constant $\delta_{f}>0$ depending only on $f$ such that for any subinterval $K$ of $J$

$$
\delta_{f} \frac{\lambda\left(f^{k}(K)\right)}{\lambda(K)}<\frac{\lambda\left(f^{k}(J)\right)}{\lambda(J)} .
$$

Proof. Take $x_{0}, y_{0}$ belonging to $\operatorname{Int}(J)$ such that

$$
\left|\left(f^{k}\right)^{\prime}\left(x_{0}\right)\right|=\frac{\lambda\left(f^{k}(J)\right)}{\lambda(J)}, \quad\left|\left(f^{k}\right)^{\prime}\left(y_{0}\right)\right|=\frac{\lambda\left(f^{k}(K)\right)}{\lambda(K)} .
$$

From $f \in \mathscr{L}(I)$ one gets

$$
\begin{aligned}
|\log |\left(f^{k}\right)^{\prime}\left(y_{0}\right)|-\log |\left(f^{k}\right)^{\prime}\left(x_{0}\right) \| & \leq \sum_{i=0}^{k-1}|\log | f^{\prime}\left(f^{i}\left(y_{0}\right)\right)|-\log | f^{\prime}\left(f^{i}\left(x_{0}\right)\right) \| \\
& <\sigma \sum_{i=0}^{k-1} \lambda\left(f^{i}(J)\right)
\end{aligned}
$$

for an approximate $\sigma$, but it is obvious that $\lambda\left(f^{k-j}(J)\right)<\lambda(I) / \kappa_{f}^{j-1}$ for any $1 \leq j \leq k$. Therefore,

$$
\delta_{f}\left|\left(f^{k}\right)^{\prime}\left(y_{0}\right)\right|<\left|\left(f^{k}\right)^{\prime}\left(x_{0}\right)\right|
$$

where $\delta_{f}=\exp \left(-\sigma \lambda(I) \kappa_{f} /\left(\kappa_{f}-1\right)\right)$. From this, Lemma 3.3 follows.

Remark 3.4. Lemma 3.3 does not hold if functions $\left(f_{i}\right)^{\prime}$ (see Definition 1.7) are only absolutely continuous. For a counterexample, see [12]. 
Consider $\varepsilon_{f}$ from Proposition 3.1. Fix a family $\bar{I}_{1}, \ldots, \bar{I}_{l(f)}$ of subintervals of $I$ such that for any subinterval $J$ of $I$ with $\lambda(J) \geq \varepsilon_{f}$ there exist some $i \in\{1, \ldots, l(f)\}$ holding $\bar{I}_{i} \subset J$. Let $\gamma_{f}>0$ such that if $J$ and $\bar{I}_{i}$ are in these conditions, $\lambda\left(\bar{I}_{i}\right)>\gamma_{f} \lambda(J)$.

Lemma 3.5. Let $f \in \mathscr{L}(I)$. Then $\lambda\left(\bigcup_{n=0}^{\infty} f^{-n}(\mathscr{O}(f))\right)=\lambda(I)$.

Proof. First we show that for any subinterval $J$ of $I$ there exists $k$ such that $f^{k}(J) \cap \operatorname{Int}(\mathscr{O}(f)) \neq \varnothing$. Indeed assume that $\operatorname{Cl}\left(\bigcup_{n \geq 0} f^{n}(J)\right) \cap \operatorname{Int}(\mathscr{O}(f))=\varnothing$. Since $f$ is expanding, $\operatorname{Cl}\left(\bigcup_{n \geq 0} f^{n}(J)\right)=K_{1} \cup \cdots \cup K_{l}$ with the $K_{i}$ pairwise disjoint closed intervals. There exists then a positive number $m$ such that for some $i_{0} \in\{1, \ldots, l\}, f^{m}\left(K_{i_{0}}\right) \subset K_{i_{0}}$. Now $\left.f^{m}\right|_{K_{i_{0}}}: K_{i_{0}} \rightarrow K_{i_{0}}$ is an expanding function, so by Lemma 2.7 it has a bitransitive interval, which is impossible.

In particular, for every $\bar{I}_{i}$ there exist $m(i)$ and $\xi>0$ such that $\lambda\left(\bar{I}_{i} \cap f^{-m(i)}(\mathscr{O}(f))\right)>\xi \lambda\left(\bar{I}_{i}\right)$, with $\xi$ not depending on $i$.

Note that to prove Lemma 3.5 it is sufficient to show that if $J$ is a subinterval of $I$ there exist a constant $\rho>0$ not depending on $J$ and a finite collection $J_{1}, \ldots, J_{r}$ of pairwise disjoint subintervals of $J$ verifying $\bigcup_{i=1}^{r} J_{i} \subset$ $\bigcup_{n=0}^{\infty} f^{-n}(\mathscr{O}(f))$ and $\sum_{i=1}^{r} \lambda\left(J_{i}\right)>\rho \lambda(J)$. But this can easily be obtained from Proposition 3.1 and Lemma 3.3 by taking $\rho=\xi \gamma_{f} \delta_{f}$.

Remark 3.6. A similar result to Lemma 3.5 was proved in [16] by means of techniques related with the Frobenius-Perron operator.

The next lemma generalizes Lemma 1 from [20].

Lemma 3.7. Let $f \in \mathscr{L}(I)$ be bitransitive. Suppose $J$ is a subinterval of $I$ and $M$ an infinite set of nonnegative integers. Then there exists a Borel set $B_{f}(J, M) \subset I$ such that $\lambda\left(B_{f}(J, M)\right)=\lambda(I)$ and with the property that for any $x \in B_{f}(J, M)$ there exist infinitely many $n \in M$ with $f^{n}(x) \in J$.

Proof. For any $j$ let $M_{j}=\{n \in M: n \geq j\}$ and $B_{j}=\left\{x \in I: f^{k}(x) \in J\right.$ for some $\left.k \in M_{j}\right\}$. If we show that $\lambda\left(B_{j}\right)=\lambda(I)$, obviously $B_{f}(J, M)=\bigcap_{j} B_{j}$ will be the desired set.

Since $f \in \mathscr{L}(I)$, it follows from [24] that it has an invariant probability measure $\mu$ absolutely continuous with respect to $\lambda$. We can suppose $\mu(I)=$ $\lambda(I)$. From Lemma 2.3 it follows that there exists $s(f)$ not depending on $i$ such that $f^{s(f)}\left(\bar{I}_{i}\right)=I$ for every $i$. Thus a similar argument as in Lemma 3.5 gives $\lambda\left(\bigcup_{n=0}^{\infty} f^{-n}(J)\right)=\lambda(I)$, which also implies by the absolute continuity of $\mu$ that $\mu\left(\bigcup_{n=0}^{\infty} f^{-n}(J)\right)=\lambda(I)$. By $\mu$ being invariant, $\mu\left(f^{-k}(J)\right)=\mu\left(f^{-l}(J)\right)>$ 0 for any $k$ and $l$, and by the continuity of $\mu$ again $\inf _{n}\left\{\lambda\left(f^{-n}(J)\right)\right\}>0$. Therefore, there exists $\eta>0$ not depending on $i$ such that, for any $m \geq s(f)$, $\lambda\left(\bar{I}_{i} \cap f^{-m}(J)\right)>\eta \lambda\left(\bar{I}_{i}\right)$. Now we can show $\lambda\left(B_{j}\right)=\lambda(I)$ in an analogous way as in Lemma 3.5.

Lemma 3.8. Let $f \in \mathscr{L}(I)$ and $J, K$ be bitransitive intervals of $f$ such that $J \cap K \neq \varnothing$. Suppose $L_{1}, L_{2}$ are subintervals of $I$ such that $f^{l}\left(L_{1}\right) \subset J$, $f^{\prime}\left(L_{2}\right) \subset K$ for some nonnegative integer $l$. Then $\lambda^{2}\left(\mathrm{Ch}(f) \cap\left(L_{1} \times L_{2}\right)\right)=$ $\lambda\left(L_{1}\right) \lambda\left(L_{2}\right)$.

Proof. It is not restrictive to suppose $l=0, L_{1}=J, L_{2}=K$. On the other hand, from Lemma 2.5 (ii) we get that either $J=K$ or $J \cap K=\{a\}$. Assume that we are in the second case (the first one is analogous). 
For example, suppose that $g=\left.f^{r}\right|_{J}, h=\left.f^{s}\right|_{K}$ are bitransitive, and $K=$ $[a, b]$. Using Lemma 3.7 and reasoning as in [20], one can find a Borel set $A \subset J$ with $\lambda(A)=\lambda(J)$ such that for any $x \in A$

$$
\limsup _{n \rightarrow \infty} g^{n !}(x)=a .
$$

Take $x \in A$. Let $(r(n))_{n=1}^{\infty}$ be an increasing sequence of multiplies of $r s$ with $\left(f^{r(n)}(x)\right)_{n=1}^{\infty}$ converging to $a$, and put $M=\{r(n) / s\}_{n=1}^{\infty}$. Also choose sequences $\left(K_{n}\right)_{n=1}^{\infty}$ and $\left(K^{n}\right)_{n=1}^{\infty}$ of subintervals of $K$ containing respectively $a$ and $b$ such that their lengths tend to zero. Then Lemma 3.7 gives that

$$
A(x)=\bigcap_{n=1}^{\infty}\left(B_{h}\left(K_{n}, M\right) \cap B_{h}\left(K^{n}, M\right)\right)
$$

has measure $\lambda(K)$. Since for every $x \in A$ and every $y \in A(x)$

$$
\limsup _{n \rightarrow \infty}\left|f^{n}(x)-f^{n}(y)\right|>0, \quad \liminf _{n \rightarrow \infty}\left|f^{n}(x)-f^{n}(y)\right|=0,
$$

Lemma 3.8 follows.

Proof of Theorem 1.8. It is a direct consequence of Lemmas 2.5, 3.5, 3.8, and 2.3.

Remark 3.9. With the same argument as in [20] one can also prove that any $f \in \mathscr{L}(I)$ possesses a scrambled set of positive exterior measure.

\section{ACKNOWLEDGMENT}

I am grateful to the referee for many useful remarks on the first version of this paper.

\section{REFERENCES}

1. F. Balibrea Gallego and V. Jiménez López, A structure theorem for $C^{2}$ functions verifying the Misiurewicz condition, Proceedings of the European Conference on Iteration Theory (ECIT 91), Lisbon, 1991 (to appear).

2. M. Barge and J. Martin, Chaos, periodicity, and snakelike continua, Trans. Amer. Math. Soc. 289 (1985), 355-365.

3. A. Buckner and T. Hu, On scrambled set and chaotic functions, Trans. Amer. Math. Soc. 301 (1987), 289-297.

4. E. Coven and I. Mulvey, Transitivity and the centre for maps of the circle, Ergodic Theory Dynamical Systems 6 (1986), 1-8.

5. A. Denjoy, Sur les courbes définie pas les équations differentielles á la surface du tore, J. Math. Pures Appl. (9) 11 (1932), 333-375.

6. T. Gedeon, Generic chaos can be large, Acta Math. Univ. Comenian. 54-55 (1988), 237-241.

7. R. Graw, On the connection between periodicity and chaos of continuous functions and their iterates, Aequationes Math. 19 (1979), 277-278.

8. J. Guckenheimer, Sensitive dependence on initial conditions for one-dimensional maps, Comm. Math. Phys. 70 (1979), 133-160.

9. K. Janková and M. Polakovič, Transitivity of expanding maps of the interval, Acta Math. Univ. Comenian. 56-57 (1989), 243-247.

10. K. Janková and J. Smítal, A characterization of chaos, Bull. Austral. Math. Soc. 34 (1986), 283-292. 
11. V. Jiménez, Large chaos in smooth functions of zero topological entropy, Bull. Austral. Math. Soc. 46 (1992), 271-285.

12. V. Jiménez López, Is Liand Yorke's definition a good tool to measure chaos?, Ph.D. thesis, Univ. de Murcia, 1992.

13. I. Kan, A chaotic function possessing a scrambled set of positive Lebesgue measure, Proc. Amer. Math. Soc. 92 (1984), 45-49.

14. M. Kuchta and J. Smítal, Two point scrambled set implies chaos, European Conference on Iteration Theory (ECIT 87), World Sci. Publishing Co., Singapore, 1989, pp. 427-430.

15. T. Li and J. Yorke, Period three implies chaos, Amer. Math. Monthly 82 (1975), 985-992.

16. _ Ergodic transformations from an interval into itself, Trans. Amer. Math. Soc. 235 (1978), 183-192.

17. M. Misiurewicz, Chaos almost everywhere, Iteration Theory and its Functional Equations, Lecture Notes in Math., vol. 1163, Springer, Berlin, 1985, pp. 125-130.

18. J. Piórek, On the generic chaos in dynamical systems, Acta Math. Univ. Iagell. 25 (1985), 293-298.

19. Ľ. Snoha, Generic chaos, Comment. Math. Univ. Carolin. 31 (1990), 793-810.

20. J. Smital, A chaotic function with some extremal properties, Proc. Amer. Math. Soc. 87 (1983), (54-56).

21. A chaotic function with a scrambled set of positive Lebesgue measure, Proc. Amer. Math. Soc. 92 (1984), 50-54.

22. Chaotic functions with zero topological entropy, Trans. Amer. Math. Soc. 297 (1986), 269-282.

23. S. van Strien, Smooth dynamics on the interval, New Directions in Dynamical Systems, Cambridge Univ. Press, Cambridge, 1988, pp. 57-119.

24. S. Wong, Some metric properties of piecewise monotonic mappings of the unit interval, Trans. Amer. Math. Soc. 246 (1978), 493-500.

Departamento de Matemáticas, Universidad de Murcia, Campus de Espinardo, Aptdo. De Correos 4021, 30100 Murcia, Spain

E-mail address: tesquembetc.um.es 